\title{
Advanced control of low order modes in laser guide star multi-conjugate adaptive optics systems
}

Carlos Correia, Jean-Pierre Véran, Glen Herriot, Brent Ellerbroek, Lianqi Wang, et al.

Carlos Correia, Jean-Pierre Véran, Glen Herriot, Brent Ellerbroek, Lianqi Wang, Luc Gilles, "Advanced control of low order modes in laser guide star multi-conjugate adaptive optics systems," Proc. SPIE 8447, Adaptive Optics Systems III, 84471S (13 September 2012); doi: 10.1117/12.926872

Event: SPIE Astronomical Telescopes + Instrumentation, 2012, Amsterdam, Netherlands 


\title{
Advanced control of low order modes in laser guide star multi-conjugate adaptive optics systems
}

\author{
Carlos Correia $^{a}$, Jean-Pierre Véran ${ }^{a}$, Glen Herriot ${ }^{a}$, \\ Brent Ellerbroek $^{b}$, Lianqi Wang ${ }^{b}$ and Luc Gilles ${ }^{b}$ \\ ${ }^{a}$ National Research Council, Herzberg Institute of Astrophysics, \\ 5071 W. Saanich Rd, Victoria, BC, V9E 2E7, Canada \\ $b$ Thirty Meter Telescope Observatory Corporation, \\ Suite 200, 1111 S. Arroyo Parkway, Pasadena CA, USA
}

\begin{abstract}
Laser-guide-star-based multi-conjugate adaptive optics (MCAO) systems require natural guide-stars to measure tilt and tilt-anisoplanatism modes.

This paper focuses on the parameter optimisation of sub-optimal integrator-based controllers using a single and a double integrator (baseline option) to drive the low-order loop of NFIRAOS, the 1st light MCAO system for the Thirty-Meter Telescope. The minimum-variance (MV) controller is outlined, against which integrators are compared.

Simulations using $~ 500$ asterisms considered in sky-coverage simulations for the TMT show that the double integrator gives competitive results thoughout the range of asterisms and magnitudes considered. It is shown that using an optimal modal gain integrator can further improve the performance with respect to using an averaged gain for all of part of the modes. However, it is outperformed by the MV controller, in particular for asterisms with relatively dim stars (lower bound is magnitude 22 in H-band) requiring low temporal frame-rates (as low as $16 \mathrm{~Hz}$ ) to integrate more flux. Over all the cases tested, an average of $\sim 100 \mathrm{~nm} \mathrm{rms}$ (23 $\mathrm{nm}$ rms median) improvement can be achieved with the MV. The MV further increases by $15 \%$ the probability of working below the 50th-percentile residual of the double integrator.
\end{abstract}

Keywords: Minimum-variance reconstruction, tilt anisoplanatism, Kalman filtering, Atmospheric tomography

\section{INTRODUCTION}

Adaptive Optics (AO) systems are used to recover the angular resolution of ground-based telescopes by correcting in real-time the wave-front disturbances introduced during propagation across the Earth's atmosphere. ${ }^{1,2}$ The classical concept with one wave-front sensor (WFS) and one deformable mirror (DM) is limited by angular anisoplanatism, thus only providing correction over a small field-of-view.

In order to correct the atmosphere over a larger patch, tomography or volumetric estimation of the wavefront above the observatory is required. For that, several wave-front sensors (WFS) are used to measure the atmospheric disturbances in different directions. Those measurements are then utilised to estimate the threedimensional wave-front phase distortions on a discrete number of layers in the atmosphere based on a priori spatial second-order moments of the of the wave-front phase and measurement noise. Such algorithms are called static minimum-variance (MV) since they minimise the residual pupil-integrated phase variance - the same is to say they optimise the Strehl-ratio ${ }^{3}$ - and do not take any temporal dynamics into account. Finally, for a multi-conjugate system $(\mathrm{MCAO})$ the phase estimates are projected onto several $(>1)$ deformable-mirrors $(\mathrm{DM})$ conjugated to different ranges, thus providing a correction across a much larger field. ${ }^{4}$

However, it has soon been realised that only using natural guide-stars to drive the AO systems would reach very small portions of the available sky, even on future generation of 30 to 40m-class Extremely Large Telescopes

Send correspondence to Carlos Correia carlos.correia@nrc-cnrc.gc.ca

Adaptive Optics Systems III, edited by Brent L. Ellerbroek, Enrico Marchetti, Jean-Pierre Véran, Proc. of SPIE Vol. 8447, 84471 S · c 2012 SPIE · CCC code: $0277-786 / 12 / \$ 18 \cdot$ doi: $10.1117 / 12.926872$ 
(ELT). It became clear that laser guide stars (LGSs) will be required to achieve scientifically useful levels of sky-coverage for astronomical MCAO systems.

Unlike natural guide-star MCAO, laser-tomography MCAO suffers from tilt anisoplanatism. This so occurs because single LGS cannot be used to measure wave-front tilt since, being launched from the ground and backscattered high in the atmosphere, the precise position of the laser beacon on the sky is uncertain - an effect due to the upwards and downwards beam propagating across the same atmospheric path. Range variations make the absolute focus measurement impossible to obtained with LGS, but this case is not dealt with here.

With multiple LGS, tilt anisoplanatism arises from a combination of a few modal components of the atmospheric profile. These components are a combination of quadratic wave-front aberrations that produce fielddependent tip/tilt (TT), which cannot be measured by the LGS WFS. Throughout this document, NGS modes refers to the 5 modes comprising TT and TT-anisoplanatism (TTA), the latter being also called plate-scale modes, in analogy to deformations given by the solutions to classical plate equations.

\section{TMT $1^{\text {st }}$-light facility Narrow-Field Infrared Adaptive Optics System}

The Thirty-Meter Telescope (TMT) $1^{\text {st }}$-light facility Narrow-Field Infrared Adaptive Optics System (NFIRAOS $)^{5,6}$ is a MCAO system with two deformable mirrors (DMs) conjugated to the range of $0 \mathrm{~km}$ and 11.2 $\mathrm{km}$, respectively, an asterism of 6 sodium laser guide stars arranged in a pentagon with a 35" radius plus one more on-axis, and up to three NIR ( $\mathrm{J}$ and $\mathrm{H}$ band) on-instrument natural guide star low-order wavefront sensors (OIWFS).

High sky-coverage goals will require considerably dim NGS down to magnitude 22 in H-band, which implies increasing the exposure times to integrate sufficient light flux. Thus, the NGS loops may run at low frame-rates even with the light-gathering capacity of the ELTs. NFIRAOS is expected to run at [20-800] Hz with $90 \mathrm{~Hz}$ median to meet the tight sky-coverage specification of $50 \%$ at the galactic pole. ${ }^{7}$ The overall control scheme adopted here is split tomography, in which the laser and natural guide star loops are driven independently, ${ }^{8}$ though a better performing jointly-optimal scheme has been proposed in [Gilles et al, 2009]. ${ }^{9}$

This paper revisits the tilt-anisoplanatism principles inSect. 2 and focuses on the optimisation of the integrator-based controller parameters in Sect. 3 where a optimal modal gain integrator is proposed. The MV controller is outlined in Sect. 4. Numerical results are presented in Sect. 5.

\section{LINEAR MODELLING OF TT/TTA MODES}

\subsection{TT/TTA-modes geometry}

Most of the error in the TTA modes can be corrected for by applying a combination of three quadratic Zernike modes with proportional amplitudes in two conjugate planes. ${ }^{10}$ For a two DM MCAO system like the TMT NFIRAOS, 5 plate scale modes carry over $80 \%$ of the total error variance due to tilt anisoplanatism. These are the global TT modes and the three dominant tilt anisoplanatism modes, a combination of focus and astigmatisms, that must be measured using the low order NGS WFS.

The wave-front that cannot be seen by the LGS WFS takes the form

$$
\varphi(\rho, \theta, t)=\sum_{j=2}^{6} \alpha_{j}(t) Z_{j}\left(\frac{\rho}{R}\right)+\sum_{j=4}^{6} \beta_{j}(t) Z_{j}\left(\frac{\rho+\theta h_{c}}{R_{h}}\right),
$$

where $\alpha_{j}$ and $\beta_{j}$ are the Zernike coefficients (following the ordering of Noll ${ }^{11}$ ) in the lower and upper DMconjugate planes. For NFIRAOS the conjugate range of the upper DM in kilometres is $h_{c}=11.2 \mathrm{Km}, R$ is the radius of the telescope and $R_{h}$ the radius of the meta-pupil at $h=h_{c}$.

The coefficients $\beta_{j}$ can be worked out provided $\varphi(\rho, \theta, t)$ is such that the resultant only contains fielddependent TT to which the LGS-WFS are insensitive. The relation is given by $\beta_{j}=-r_{l}^{2} \alpha_{j}$, with $r_{l}$ given by the ratio of the cone-intersected pupil and meta-pupil in the DM11.2 conjugate range is (see Fig. 1 for a visual depiction)

$$
r_{l}=r_{n}\left(1-\frac{h_{c}}{h_{N a}}\right)
$$


where $r_{c} \triangleq 1-h_{c} / h_{N a}$ is the shrinking factor of the cone-intersected meta-pupil with respect to the cylinderintersected meta-pupil at $11.2 \mathrm{~km}$, translating the cone effect for a DM conjugated to range $h_{c}$ and an LGS at range $h_{N a}$ (see Fig. 1 ) and

$$
r_{n} \triangleq \frac{D}{D+F o V \times h_{c} \times 1000},
$$

normalises the upper modal coefficient to the particular choice of underlying pupils over which the modes are defined, with $F o V$ the field-of-view in radians.

The wave-front $\varphi$ is defined over 2 layers in the volume. Let

$$
\varphi(\rho, \theta, t) \triangleq\left[-\underline{\varphi}_{0}-\right](\rho, \theta, t)
$$

be a concatenation of phase coefficients of the decomposition of the WF phase at the DM-conjugate altitudes onto an orthonormalised Zernike basis. ${ }^{11}$ For the NGS modes model, only modes $Z_{2 \cdots 6}$ are used that correspond to the TT and the quadratic modes of Eq. (1), i.e. $\varphi_{k}$ is a 10 -coefficient column vector .

Expressing the Zernike polynomials using cartesian coordinates the 5 NGS modes are hence defined

$$
\begin{aligned}
x_{k}^{\text {tip }} & =2\left(x_{0}, 0\right) ; \\
x_{k}^{\text {tilt }} & =2\left(0, y_{0}\right) ; \\
x_{k}^{\Delta F} & =2 \sqrt{3}\left(\left[x_{0}^{2}+y_{0}^{2}\right] ;-\left[x_{c}^{2}+y_{c}^{2}\right] / r_{l}^{2}\right) ; \\
x_{k}^{\Delta A_{0}} & =\sqrt{6}\left(\left[x_{0}^{2}-y_{0}^{2}\right] ;-\left[x_{c}^{2}-y_{c}^{2}\right] / r_{l}^{2}\right) ; \\
x_{k}^{\Delta A_{45}} & =2 \sqrt{6}\left(\left[x_{0} y_{0}\right] ;-\left[x_{c} y_{c}\right] / r_{l}^{2}\right) ;
\end{aligned}
$$

where $x_{0}, y_{0}$ and $x_{c}, y_{c}$ are the actuator coordinates on the ground and upper DMs, normalised by the telescope radius $R$. The notation $\Delta F$ is used to denote differential focus, resulting in radial amplification in the image-plane whereas $\Delta A_{0}$ and $\Delta A_{45}$ is used for two differential amplification modes.

The relation of Zernike polynomials' coefficients to NGS mode coefficients is straightforward: the projection matrix of the 5 NGS modes (TT+TTA) onto the quadratic modes is given by (in matrix format)

$$
\vec{\varphi}_{k}^{\text {tur }} \triangleq P_{\mathrm{M} 2 \mathrm{Z}} \vec{\chi}_{k}
$$

where $P_{\mathrm{M} 2 \mathrm{Z}} \in \mathbb{R}^{10 \times 5}$ translates 5 NGS mode coefficients to their layered version expressed in Zernike polynomials.

The global tip/tilt mode vectors $x^{\text {tip }}$ and $x^{\text {tilt }}$ have the tip/tilt Zernike modes applied to the ground DM only.

\subsection{Measurement model}

In order to exactly estimate the TT and the quadratic modes that produce field-dependent TT on the LGS sensing directions, 3 independent measurements of TT in the field are required. ${ }^{10}$ In the particular case of NFIRAOS, there are two single-aperture TT WFS and a $2 \times 2$ sub-aperture WFS for a third measurement of TT, defocus and two astigmatisms (TTFA).

Let the resulting aperture-plane wave-front (WF) in direction $\theta$

$$
\phi_{k}^{\mathrm{tur}}=P_{\theta} \varphi_{k}^{\mathrm{tur}},
$$

where $P_{\theta}$ is a projection matrix of Zernike polynomials on the intersected meta-pupils in DM-conjugated planes with a pupil-sized cylinder of diameter $D_{0}$ in the directions $\theta$ of the NGS. ${ }^{12}$

Since three NGS are required to estimate 5 NGS modes, matrix $P_{\theta}$ is subdivided into 3 horizontal slabs for the three OIWFS and two vertical slabs, for the two DM-conjugate altitudes.

Assume the following measurement model 


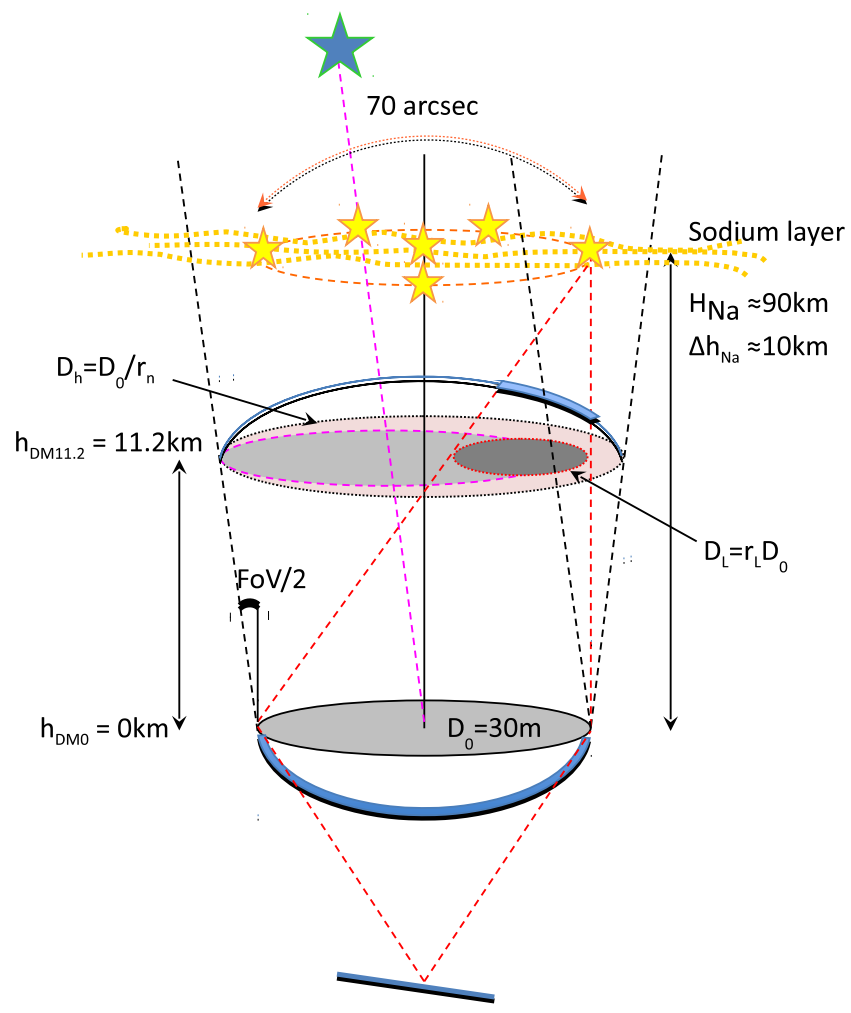

Figure 1. The tilt anisoplanatism modes each contain a quadratic Zernike polynomial with proportional amplitudes on the ground and upper DMs, whose combined effect produces pure tip/tilt in the LGS wavefront sensors (thus not sensed), but contains field dependent tip/tilt (plate scale effect) and quadratic wavefront aberrations across the science field and NGS patrol field.

$$
\begin{aligned}
s_{k}(\theta) & =\int_{(k-1) T_{s}}^{k T_{s}} \mathrm{G}\left(P_{\theta} \varphi(\tau)^{\mathrm{tur}}-P_{\theta} \varphi(\tau)^{\mathrm{cor}}\right) \mathrm{d} \tau+\eta_{k} \\
& =\mathrm{G} P_{\theta}\left(\bar{\varphi}_{k}^{\text {tur }}-\bar{\varphi}_{k}^{\text {cor }}\right)+\eta_{k} \\
& =\mathrm{G} P_{\theta} \bar{\varphi}_{k}^{\text {res }}+\eta_{k},
\end{aligned}
$$

where $\mathrm{G}$ is the wave-front-to-measurements matrix and 'tur', 'cor' and 'res' stand respectively for turbulent, correction and residual phase and $T_{s}$ is the OIWFS integration period. $\Sigma_{\eta}$ is the noise covariance matrix expressed in measurement space, i.e. a 12x12 diagonal matrix. A bar atop the wave-front phase vectors represents average over time.

For the Hartmann-Shack WFS, $s_{k}$ are the WFS slopes.

The correction phase applied by the DM is $\bar{\varphi}_{k}^{\text {cor }}=\mathrm{N} u_{k-1}$ with $\mathrm{N}$ the commands-to-phase matrix and $\eta_{k}$ is a zero-mean Gaussian-distributed spectrally white noise with $\eta \sim \mathcal{N}\left(0, \Sigma_{\eta}\right)$.

The modal matrix $\mathrm{G}$ translates modal coefficients of TT, TT and TTFA modes into average slopes over the illuminated sub-region of each sub-aperture. It is thus a contatenation of three phase-to-slopes matrices for each individual OIWFS.

Figure 2 depicts schematically how the measurements are acquired in the simulation. The vector $\phi^{\text {res }}(\rho, t) \in$ $\Re^{5 \times 1}$ has the coefficients of the first (piston-removed) Zernike polynomials. Vector $s_{k} \in \Re^{12 \times 1}$ has the averaged slopes over each OIWFS sub-aperture and over the integration time $T_{s}$. 


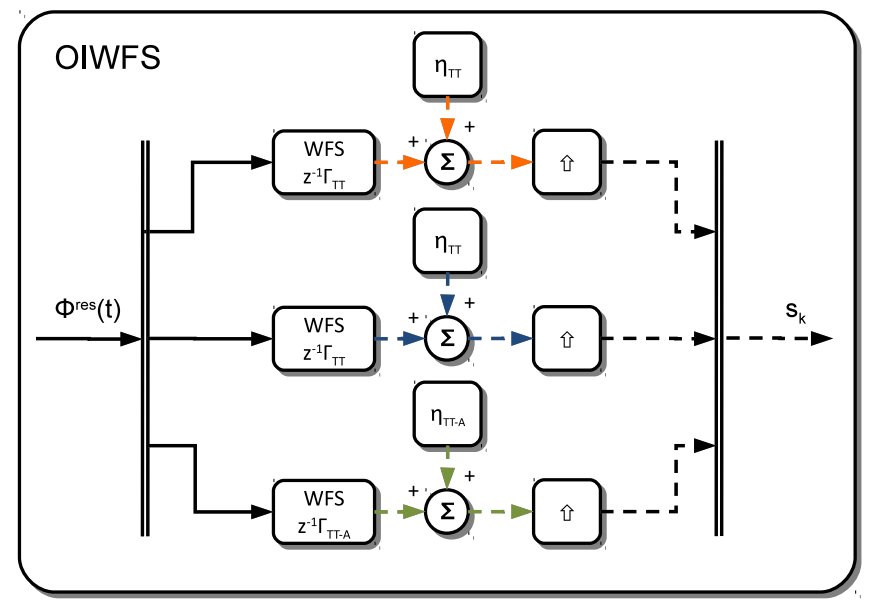

Figure 2. Block diagram of the 3 OIWFS with additive measurement noise (photon +read-out). Different coloured lines are for potentially different sampling rates. The upwards pointing arrow is used for a $z$ oh up-sampler of factors $\{1, \cdots, 40\}$. Solid-lines are used for continuous-time variables whereas dashed-lines are used for discrete-time variables.

Define

$$
P_{\odot} \triangleq P_{\theta} P_{\mathrm{M} 2 \mathrm{Z}}
$$

the projection of NGS modes onto TT and quadratic Zernike polynomials, the modes to be measured by the 3 OIWFSs. Equation (8) can be re-written as follows

$$
s_{k}(\theta)=\mathrm{G}\left(P_{\odot} \bar{\chi}_{k}^{\text {tur }}-P_{\odot} \bar{\chi}_{k}^{\text {cor }}\right)+\eta_{k}
$$

where the NGS modes are given by $\bar{\chi}_{k} \triangleq P_{\mathrm{M} 2 \mathrm{Z}}^{\dagger} \bar{\varphi}_{k}$ with $P_{\mathrm{M} 2 \mathrm{Z}}^{\dagger}$ the generalised inverse of matrix $P_{\mathrm{M} 2 \mathrm{z}}$ in Eq. (6).

\subsection{Noise model}

In the following, the noise model detailed in ${ }^{13}$ is used. It is assumed that spots are diffraction limited. Therefore, these equations apply for a Nyquist-sampled spot, i.e. with $2 \times 2$ pixels, by other words a quadrant detector.

The noise added to each sub-aperture measurement is given by (in angle rms units)

$$
\sigma_{\eta}=\frac{\theta_{b}}{\mathrm{SNR}}
$$

where $\theta_{b}$ is the effective spot size of the sub-aperture, and SNR is the signal-to-noise ratio of a single sub-aperture. For a quadrant detector, the SNR is given by

$$
\mathrm{SNR}=\frac{N_{p}}{\sqrt{N_{p}+4 N_{b}+4 \sigma_{e}^{2}}},
$$

where $N_{p}$ is the number of photo-detection events per sub-aperture, $N_{b}$ is the number of background photodetection events per sub-aperture, and $\sigma_{e}$ is the rms detector read noise per pixel.

In the IR ( $\mathrm{H}$ band), the NGS images are assumed to contain a diffraction-limited core, for which case the effective spot size is given by

$$
\theta_{b}=\frac{3 \pi \lambda \sqrt{N_{s a}}}{16 D_{0}},
$$

where $N_{s a}$ is the total number of sub-apertures for the NGS WFS. The $2 \times 2$ NGS WFS is therefore noisier than any of the two single sub-aperture NGS WFS. Note $\theta_{b}$ is twice that of the latter, since $N_{s a}$ is 4 instead of 1 and that the number of photo-detections per sub-aperture is also cut by a factor of 4 . 

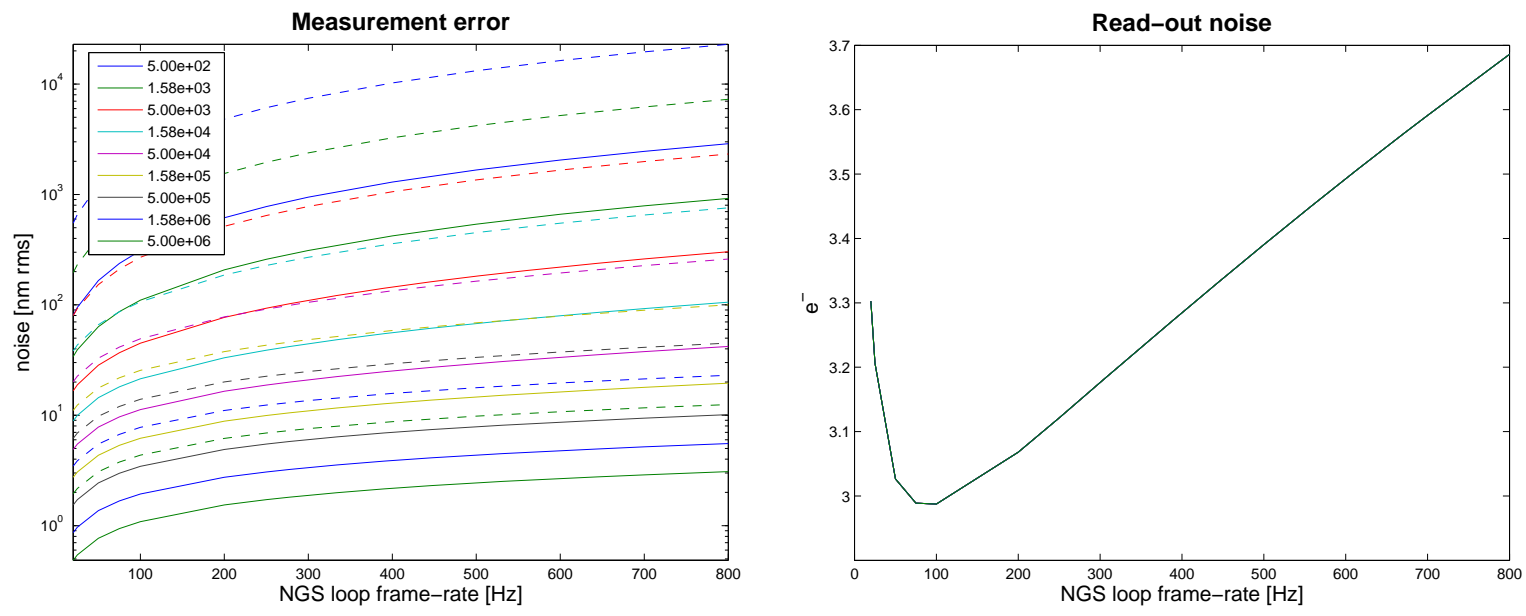

Figure 3. Left: Measurement noise error (photon-noise + read-out noise). Solid-lines: single sub-aperture OIWFS, dashed-lines: 2x2 TTF OIWFS. The noise ratio in rms units between the $2 \times 2$ and the single sub-aperture OIWFS is $\sigma_{\eta, 2 \times 2} / \sigma_{\eta, 1 \times 1} \in\{4.04, \cdots, 7.96\}$ for $f_{s} \in\{20, \cdots, 800\} \mathrm{Hz}$ and $N_{p} \in\left\{5 \times 10^{2}, \cdots, 5 \times 10^{6}\right\} \mathrm{ph} / \mathrm{m}^{2} / \mathrm{s}$.

Right: The read-out noise is $\sigma_{e}=\{2.99 \cdots 3.69\}$ for $f_{s}=\{20 \cdots 800\} \mathrm{Hz}$.

\section{OPTIMAL MODAL GAIN INTEGRATORS}

The current NFIRAOS baseline controller for the NGS modes is a dual integrator-based controller with a lead filter. $^{14,15}$ When using a sub-optimal integrator-based controller, the TT/TTA modes are estimated from the OIWFS measurements using a noise-weighted least-squares reconstructor. Using the measurement equation of Eq. (10) the noise-weighted least-squares reconstructor is given by

$$
W_{w}=\left(P_{\odot}^{\top} \mathrm{G}^{\top} \Sigma_{\eta}^{-1} \mathrm{G} P_{\odot}\right)^{-1} P_{\odot}^{\top} \mathrm{G}^{\top} \Sigma_{\eta}^{-1},
$$

with noise propagation expressed in the NGS modes is

$$
\sigma_{N P}^{2}=\frac{1}{5} \operatorname{trace}\left\{W_{w} \Sigma_{\eta} W_{w}^{\top}\right\}
$$

The diagonal entries of $W_{w} \Sigma_{\eta} W_{w}^{\top}$ are asterism-dependent since, depending on the star locations in the field, plate-scale propagates differently onto the TT modes. Thus, one is led to a modal gain controller optimisation where a set of optimal parameters is used mode-per-mode.

\subsection{Loop temporal dynamics in Laplace domain}

The open-loop transfer function for the double integrator (type-II) is defined as

$$
h_{\mathrm{ol}}(f)=h_{\mathrm{wfs}} h_{\mathrm{dac}} h_{\text {lag }} h_{\text {lead }} h_{\mathrm{int}}^{2},
$$

where the DM is considered to have a unitary transfer function, even when the woofer-tweeter scheme is used ${ }^{16}$ whereas the partial loop seen by the noise

$$
h_{\text {sys }}(f)=h_{\text {dac }} h_{\text {lag }} h_{\text {lead }} h_{\text {int }}^{2}=h_{\text {ol }} / h_{\text {wfs }},
$$

with the double integrator, lead filter, WFS, digital-to-analog converter and lag being given respectively by

$$
h_{\text {int }}^{2}(f)=g\left(\frac{1}{1-e^{-2 \pi f T_{s}}}\right)^{2},
$$




$$
h_{\text {lead }}(f)=\frac{1+2 \pi f T_{l}}{1+2 \pi f a T_{l}}, T_{l}=\frac{1}{2 \pi f_{c} \sqrt{a}},
$$

where $f_{c}$ is the frequency for which the $\left|h_{\mathrm{ol}}\right|_{\mid f=f_{c}}=1$.

$$
\begin{gathered}
h_{\mathrm{wfs}}=h_{\mathrm{dac}}=\frac{1-e^{-2 \pi f T_{s}}}{2 \pi f T_{s}}, \\
h_{\mathrm{lag}}=e^{-2 \pi f T_{\mathrm{lag}}},
\end{gathered}
$$

with $T_{\text {lag }}=1 \mathrm{~ms}$. For the single integrator, drop the square in Eq. (18a) and plug final result in Eq. (16).

The best set of parameters minimises the error function

$$
\left(g^{*}, T_{l}^{*}, a^{*}\right)=\underset{g, T_{l}, a}{\arg \min }\left\{\varepsilon_{i}^{2}\left(g, T_{l}, a\right)\right\}
$$

where

$$
\varepsilon_{i}^{2}\left(g, T_{l}, a\right)=\int_{-1 /\left(2 T_{s}\right)}^{1 /\left(2 T_{s}\right)}\left|\frac{1}{1+h_{\mathrm{ol}}\left(f, g, T_{l}, a\right)}\right|^{2} P S D_{\mathrm{NGS}, i}(f)+\left|\frac{h_{\mathrm{sys}}\left(f, g, T_{l}, a\right)}{1+h_{\mathrm{ol}}\left(f, g, T_{l}, a\right)}\right|^{2} P S D_{\eta, i}(f) \mathrm{d} f,
$$

is the residual error variance and $P S D_{\mathrm{NGS}, i}(f)$ the temporal power-spectral density of the NGS modes and $P S D_{\eta, i}(f)$ the measurement noise expressed on each mode, such that the one-sided integral $\int_{0}^{0.5 T_{s}^{-1}} P S D_{\eta, i}(f)=$ $\sigma_{\mathrm{NP}, i}^{2}, \forall i \in\{2, \cdots, 6\}$, where $\sigma_{\mathrm{NP}, i}^{2}$ are the diagonal entries of the bracketed matrix in (15).

\subsection{Parameter set optimisation}

In practice, the optimal gain for the type-I is found by starting with a small gain and increase it until $\varepsilon^{2}(g)$ reaches the global minimum, which is know to exist since the residual signal decreases monotonically with the gain and the noise propagation increases monotonically with the gain.

The set $\left(g^{*}, T_{l}^{*}, a^{*}\right)$ for the type-II can be found using two strategies: a) a constrained version in algorithm 1 and an unconstrained version in algorithm 2 where the optimal parameter set is found by dichotomy i.e. by building a data-cube of values of $\varepsilon^{2}\left(g, T_{l}, a\right)$ and picking the set $\left(g, T_{l}, a\right)$ that minimises the merit function.

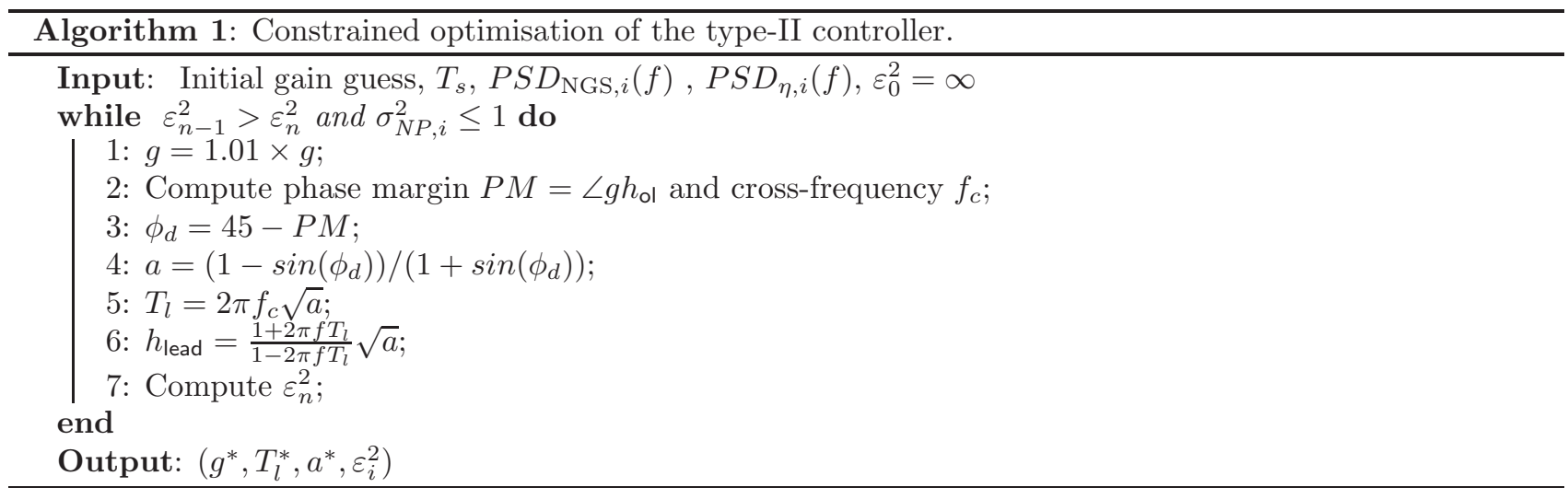

To better suit discrete-time implementation on the RTC, a discrete version of $h_{\text {lead }}$ is given by $h_{\text {lead }}(z)=$ $\left(C_{0}+z^{-1} C_{1}\right) /\left(1+D_{1} z^{-1}\right)$, with $C_{0}=\left(1+2 T_{l} / T_{s}\right) /\left(1+2 T_{l} a / T_{s}\right), C_{1}=\left(1-2 T_{l} / T_{s}\right) /\left(1+2 T_{l} a / T_{s}\right)$, and $D_{1}=\left(1-2 T_{l} a / T_{s}\right) /\left(1+2 T_{l} a / T_{s}\right)$ using bilinear approximation. 


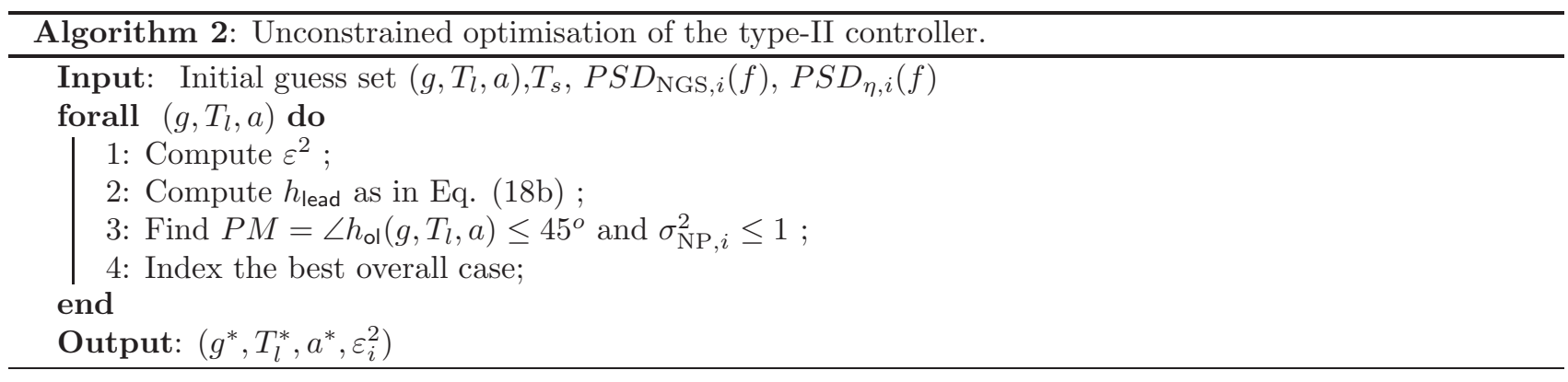

\section{MINIMUM-VARIANCE CONTROLLER}

\subsection{Minimisation of the pupil-integrated residual phase variance}

Minimising the variance of $\phi^{\text {res }}$ results in the maximisation of the Strehl-ratio (SR) ${ }^{3}$ leading to the continuoustime criterion

$$
J^{\mathrm{c}}(u) \triangleq \lim _{\tau \rightarrow+\infty} \frac{1}{\tau} \int_{0}^{\tau}\left\|\phi^{\mathrm{res}}(t)\right\|^{2} \mathrm{~d} t=\lim _{\tau \rightarrow+\infty} \frac{1}{\tau} \int_{0}^{\tau}\left\|\phi^{\mathrm{tur}}(t)-\phi^{\mathrm{cor}}(t)\right\|^{2} \mathrm{~d} t,
$$

where the residual phase is the difference between the turbulent and correction phases, $\phi^{\text {res }}(t)=\phi^{\text {tur }}(t)-\phi^{\text {cor }}(t)$ in the direction of the science targets.

Using the LQG formulation, ${ }^{17}$ the optimal negative state-feedback controller has the form

$$
u_{k}^{\mathrm{opt}}=F_{\varphi} \widehat{\bar{\varphi}}_{k+1 \mid k},
$$

where $\widehat{\varphi}_{k+1 \mid k}$ is the conditional mean of the disturbance phase to be estimated by a Kalman filter given the set of current and past measurements $\chi_{k \cdots 0}$ and $F_{\varphi}$ is a fitting operator that optimises the correction in the $\beta$-directions where are located the science-targets. Since no temporal DM-dynamics are considered, the best command is given by $u_{k}=\left(\mathrm{N}^{\top}\left\langle P_{\beta}^{\top} P_{\beta}\right\rangle_{\beta} \mathrm{N}\right)^{\dagger} \mathrm{N}^{\top}\left\langle P_{\beta} P_{\beta}^{\top}\right\rangle_{\beta} \widehat{\bar{\varphi}}_{k+1 \mid k}$ where $\langle\cdots\rangle$ represents averaging over a discrete number of directions that sample the science field. With the DM commanded directly in Zernike polynomials, i.e. $\mathrm{N}$ is the identity matrix, and noting the phase is defined at the DM-conjugate altitudes only, this simplifies to

$$
u_{k}^{\mathrm{opt}} \triangleq \widehat{\bar{\varphi}}_{k+1 \mid k} .
$$

\subsection{State space model}

Define the discrete-time state-space model

$$
\begin{aligned}
x_{k+1} & =\mathcal{A} x_{k}+\mathcal{B} u_{k}+\Gamma \varepsilon_{k} \\
s_{k} & =\mathcal{C} x_{k}+\mathcal{D} u_{k}+\eta_{k},
\end{aligned}
$$

where the state and the model matrices are a concatenation of discrete-time linear models for each of the modes (TT /TTA) - Eq. (1). The full model is hence a concatenation of individual models

$$
\begin{aligned}
x_{k} \triangleq\left(\begin{array}{c}
x_{k}^{\text {tip }} \\
x_{k}^{\text {tilt }} \\
x_{k}^{\Delta F} \\
x_{k}^{\Delta A_{0}} \\
x_{k}^{\Delta A_{45}}
\end{array}\right), & \mathcal{A} \triangleq\left(\begin{array}{cccc}
\mathcal{A}_{\text {tip }} & 0 & \cdots & 0 \\
0 & \mathcal{A}_{\text {tilt }} & \cdots & 0 \\
\vdots & \ldots & \ddots & \vdots \\
0 & \cdots & \cdots & \mathcal{A}_{\Delta F_{45}}
\end{array}\right), \\
\mathcal{B} \triangleq\left(\begin{array}{c}
\mathcal{B}_{\text {tip }} \\
\mathcal{B}_{\text {tilt }} \\
\vdots \\
\mathcal{B}_{\Delta F_{45}}
\end{array}\right), & \Gamma \triangleq\left(\begin{array}{cccc}
\Gamma_{\text {tip }} & 0 & \cdots & 0 \\
0 & \Gamma_{\text {tilt }} & \cdots & 0 \\
\vdots & \cdots & \ddots & 0 \\
0 & \cdots & \cdots & \Gamma_{\Delta F_{45}}
\end{array}\right),
\end{aligned}
$$




$$
\mathcal{C} \triangleq \mathrm{G} P_{\odot} M_{1}, \quad \mathcal{D} \triangleq-z^{-1} \mathrm{G} P_{\odot} \mathrm{N},
$$

where $M_{1}$ is a $0-1$ valued matrix that extracts the component $\bar{\chi}_{k}$, i.e. the average TT/TT-A mode over $T_{s}$. The steps to build the individual mode-by-mode model can be found in. ${ }^{17,18}$ Note that the NGS modes are (spatially) statistically independent (so are the quadratic Zernike polynomials for a Kolmogorov turbulence spectrum). The temporal correlations assuming Taylor's hypothesis of frozen flow are not considered here.

The Kalman filter is seamlessly obtained from an estimation Riccati equation. Since the metric of interest in $\mathrm{AO}$ is the long exposure integration of light on the science instruments such that $T_{\mathrm{LE}}>>T_{s}$, the asymptotic solution can be used with strictly no loss of performance. The optimal gains are computed off-line from

$$
\mathcal{L}_{\infty}=\mathcal{A} \mathcal{H}_{\infty}=\mathcal{A} \Sigma_{\infty} \mathcal{C}^{\top}\left(\mathcal{C} \Sigma_{\infty} \mathcal{C}^{\top}+\Sigma_{w}\right)^{-1}
$$

where $\Sigma_{\infty}$ is solution of the associated algebraic Riccati equation (ARE). The controller is applied in real-time by computing, at iteration $k$

$$
\begin{aligned}
\widehat{x}_{k \mid k} & =\widehat{x}_{k \mid k-1}+\mathcal{H}_{\infty}\left(s_{k}-\mathcal{C} \widehat{x}_{k \mid k-1}\right) \\
\widehat{x}_{k+1 \mid k} & =\mathcal{A} \widehat{x}_{k \mid k}+\mathcal{B} u_{k} \\
u_{k} & =\widehat{\bar{\varphi}}_{k+1 \mid k},
\end{aligned}
$$

where the hat represents conditional mean estimation of the state. The fitting matrix $F_{\varphi}$ is defined in Eq. (23).

Figure 4 shows the block diagram of the recursive implementation of Eq. (27).

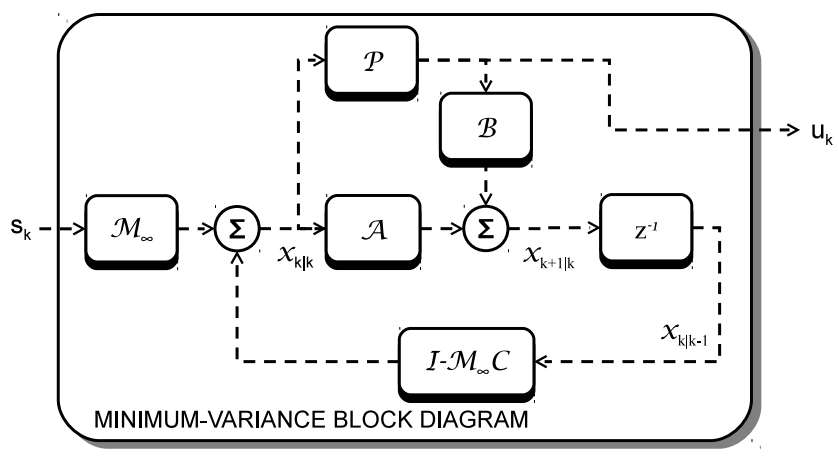

Figure 4. MV controller. Matrix $P$ is used here to compute $u_{k}$ from the estimated state $x_{k \mid k}$. With respect to Eq. (27), $u_{k}=\widehat{\bar{\varphi}}_{k+1 \mid k}=M_{\mid} x_{k \mid k}=P x_{k \mid k}$, with $M_{1}$ a 0 -1 valued matrix that extracts components $\widehat{\bar{\varphi}}_{k+1 \mid k, i}$ from the complete state.

The model of Eq. (25) is built upon the method laid out in ${ }^{17}$ with the $\mathcal{A}$ matrices identified by fitting the first steps of the temporal auto-correlation function (computed from the temporal PSDs with the Wiener-Khinchine theorem). Matrices $\Gamma$ are determined from the solution of discrete-time Lyapunov equations that define the state covariance noise from the model $\mathcal{A}$ and the total disturbance of each mode (computed from the numerical integration of the temporal PSDs). ${ }^{7}$

\subsection{Use of multiple frame-rates}

One could arguably point out that in order to further optimise the NGS loop one should pick one frame-rate per OIWFS and combine optimally the available information at each time step, as is explained in ${ }^{19}$ for the NGS/LGS case.

End-to-end simulations have been setup to test this potential alternative. However the multi-rate controller consistently failed to provide better results. The reason behind being that, at a given time-step, using the information for a reduced set of OIWFS measurements renders the reconstruction rank-deficient, for the matrix $\mathrm{G}_{\text {trunc }} P_{\odot}$ where $\mathrm{G}_{\text {trunc }}$ is matrix $\mathrm{G}$ with rows corresponding to the inexistent measurements removed, is of rank $<5$ and thus the controller fails to provide a good correction. 
Since the modes are spatially uncorrelated, uncorrelated between layers and temporally considered uncorrelated (and furthermore have similar variances and temporal spectra) the estimate produced from a reduced set using multi-rate can always be outperformed by choosing a common frequency for all the OIWFS and using a more straightforward single-rate LQG controller.

\section{CONTROLLER COMPARISON VIA FREQUENCY AND TIME-DOMAIN SIMULATIONS}

A custom code was written to compare the NGS processing with 5 NGS modes using integrator-based controllers (single and double integrators) to the MV solution. The disturbances considered consist of TT with $50 \%$ percentile wind-shake on both axis with a total of 18.6 mas rms for a median profile with $r_{0}=0.186 \mathrm{~m}, L_{0}=30 \mathrm{~m}$. The TTA modes were numerically computed using formulae in [Wang et all ${ }^{7}$ with a total of $407 \mathrm{~nm} \mathrm{rms}$ per mode. Results are computed assuming the NGS wavelength in J band using an optimal frame-rate computed on a asterism-per-asterism basis and averaged over $55 \mathrm{~s}$ of equivalent real-time simulation (excluding the initial transient period). Noise is computed assuming a Strehl-ratio variation across the 2 arcmin patrol field-of-view from 0.5 to 0.1 at the edge. Total throughput is 0.3 , a rather small value chosen to boost overall noise to compensate for aliasing and implementation errors that are not in the simulation but are considered in the full-featured sky-coverage simulations.

Figure 5 shows the residual obtained with the constrained and UNconstrained algorithms for a single mode. On the right y-axis, the phase margin obtained is roughly $65 \mathrm{deg}$ for all the temporal sampling frequencies tested, suggesting that imposing a $65 \mathrm{deg}$ phase margin instead of a $45 \mathrm{deg}$ is best in two ways: more stability and less residual. To reduce the computational burden, the constrained optimisation is used to quickly find a sweet spot around which a 2D grid of values for $a$ and $T_{l}$ is tested to further reduce the residual.

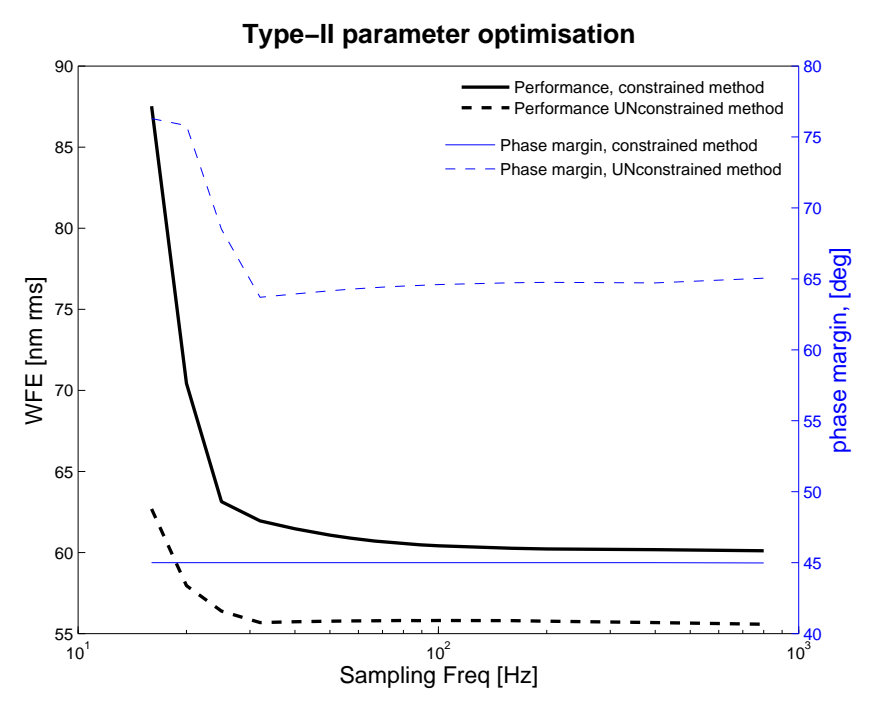

Figure 5. Constrained vs UNconstrained optimisation. In this case, for computational performance purposes, the best lead parameters where found for the best gain found using algorithm 1.

Using the re-optimisation of the type-II controller parameters as is explained in Fig. 5 results in further 43 $\mathrm{nm} \mathrm{rms}$ on average ( $3 \mathrm{~nm}$ rms median) improvement with respect to using the constrained optimisation alone. A further case was compared where the optimal parameter set is found on a mode-per-mode basis or as an averaged parameter set for the TT and the TTA modes. A small improvement is obtained with $3.6 \mathrm{~nm} \mathrm{rms}(2.4 \mathrm{~nm} \mathrm{rms}$ median) for the type-II controller whereas $48 \mathrm{~nm} \mathrm{rms}$ ( $15.5 \mathrm{~nm}$ rms median) were found for the type-I controller. The greater differences are particularly appreciated for the noisier cases at low temporal frame-rates.

Figure 6 plots the overall results obtained over 500 asterisms. On the left panel, results are given as a function of the frame-rate whereas on the right panel the cumulative residuals are plotted. In both cases the 
theoretical residual obtained using transfer functions are also given for comparison. The theoretical residual consistently under-estimates the actual residual obtained via time-domain simulations, a sign that a transferfunction approach isn't fully appropriate to describe a hybrid loop with continuous and discrete phenomena. The discrepancies are greater at very low temporal rates.

Over all the cases tested, an average of $\sim 100 \mathrm{~nm}$ rms (23 nm rms median) improvement can be achieved. The MV increases by $15 \%$ the probability of working below the 50th-percentile residual of the double integrator (baseline option). Conversely, for the 50th-percentile, the MV obtains $\sim 25 \mathrm{~nm}$ rms residual against the $\sim 35 \mathrm{~nm}$ rms of the double integrator, i.e. roughly $25 \mathrm{~nm} \mathrm{rms}$ improvement in quadrature.
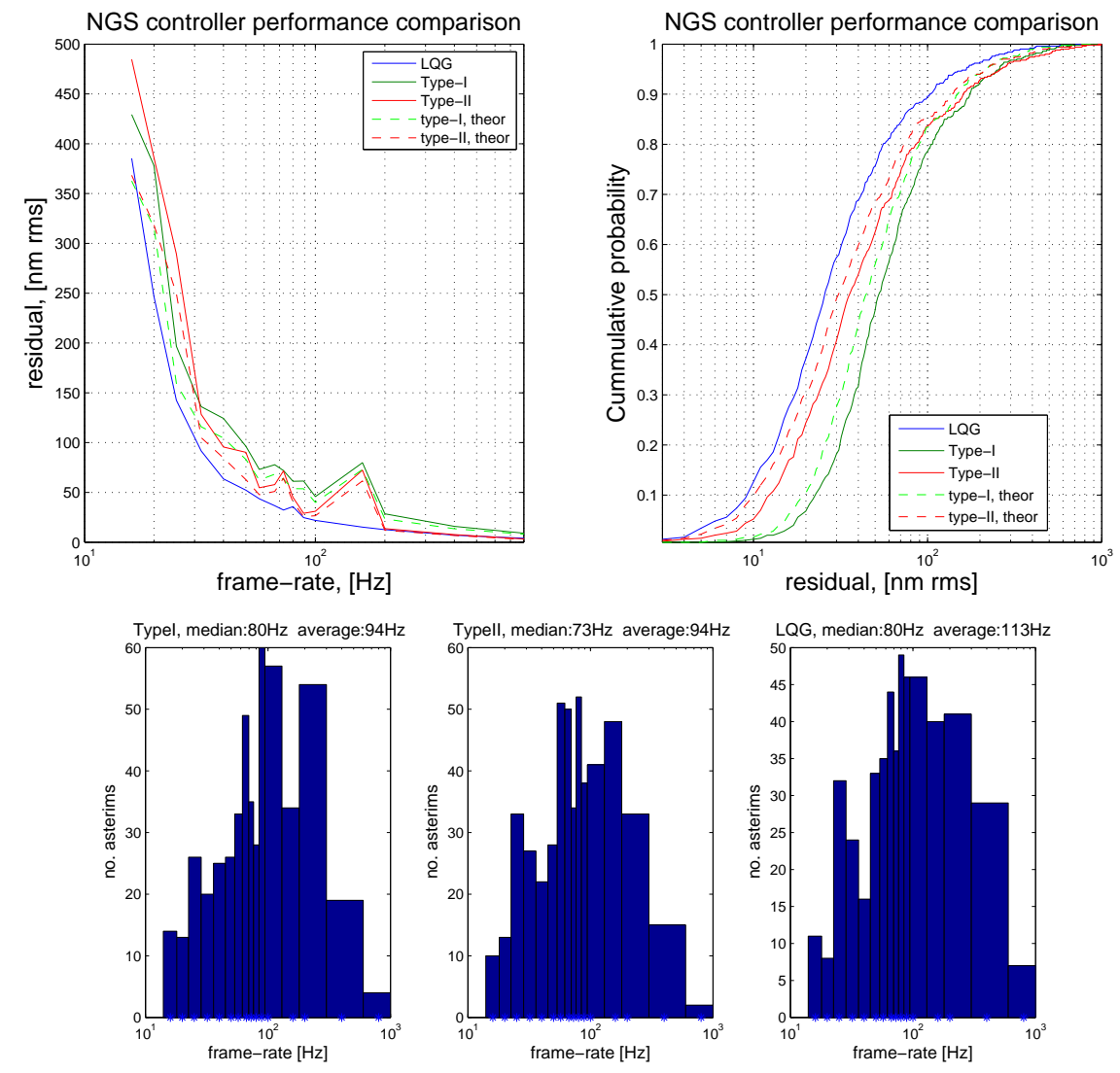

Figure 6. Performance comparison. Top panel: residuals in $\mathrm{nm}$ rms, averaged over all the cases (left) and cumulative probability (left). Bottom panel: histogram of frame-rates for the 3 controllers. The MV tends to use higher frame-rates, with a median of $80 \mathrm{~Hz}$ and average of $113 \mathrm{~Hz}$.

\section{CONCLUSION}

Using end-to-end temporal numerical simulations it has been shown that the optimal modal gain double integrator is a competitive alternative to the optimal minumum-variance controller since it is conceptually simpler and relatively straightforward to optimise. Using a more general unconstrained procedure developed here, the double integrator was further improved by $\sim 43 \mathrm{~nm} \mathrm{rms}$ on average ( $3 \mathrm{~nm} \mathrm{rms}$ median) with respect to its previous configuration.

However, the integrator-based controllers cannot reach the same levels of performance as the minimumvariance controller, in particular when only dim stars are used to probe the NGS modes (requiring low temporal frame-rates as low as $16 \mathrm{~Hz}$ ). Subtracting the residuals in quadrature, an average improvement of roughly $100 \mathrm{~nm}$ rms (23 nm rms median) was found using the MV with respect to the re-optimised double integrator. The MV 
further increases by $15 \%$ the probability of working below the 50th-percentile residual of the double integrator. This suggests that the sky-coverage can be improved by factors of $10 \%$ or more for the median case.

The advantages of the MV are manifold. Although conceptually more complex, it is much faster to compute off-line, vibration suppression can be easily embedded, supports up-sample of commands to the LGS loop framerate $\left(800 \mathrm{~Hz}\right.$ for NFIRAOS) and is directly optimised in discrete-time. ${ }^{16}$

The model will in the future support absolute focus error due to sodium range variations. The authors plan to compare the controllers using the full featured TMT sky-coverage code using the simulator MAOS *.

\section{REFERENCES}

[1] Roddier, F., [Adaptive Optics in Astronomy], Cambridge University Press, New York (1999).

[2] J.W.Hardy, [Adaptive Optics for Astronomical Telescopes], Oxford, New York (1998).

[3] Herrmann, J., "Phase variance and Strehl ratio in adaptive optics.," J. Opt. Soc. Am. A 9, 2257-2258 (Dec. 1992).

[4] Beckers, J. M., "Increasing the size of the isoplanatic patch with multiconjugate adaptive optics," in [ESO Conference on Very Large Telescopes and their Instrumentation], 2, 693-703 (oct 1988).

[5] Ellerbroek, B., Adkins, S., Andersen, D., Atwood, J., Browne, S., Boyer, C., Byrnes, P., Caputa, K., Conan, R., Cousty, R., Erikson, D., Fitzsimmons, J., Gamache, F., Gilles, L., Herriot, G., Hickson, P., Lardier, O., Morin, P., Pazder, J., Pfrommer, T., Quinn, D., Reshetov, V., Roberts, S., Sinquin, J.-C., Schoeck, M., Smith, M., Tyler, G., Vaughn, J., Veran, J.-P., Vogel, C., Wang, L., and Wevers, I., "First light adaptive optics systems and components for the Thirty Meter Telescope," Adaptive Optics Systems II 7736(1), 773604, SPIE (2010).

[6] Herriot, G., Andersen, D., Atwood, J., Boyer, C., Beauvillier, A., Byrnes, P., Conan, R., Ellerbroek, B., Fitzsimmons, J., Gilles, L., Hickson, P., Hill, A., Jackson, K., Lardière, O., Pazder, J., Pfrommer, T., Reshetov, V., Roberts, S., Véran, J.-P., Wang, L., and Wevers, I., "NFIRAOS: TMT's facility adaptive optics system," Adaptive Optics Systems II 7736(1), 77360B, SPIE (2010).

[7] Wang, L., Ellerbroek, B., and Veran, J. P., "High fidelity sky coverage analysis via time domain adaptive optics simulations," Appl. Opt. 48(27), 5076-5087 (2009).

[8] Gilles, L. and Ellerbroek, B. L., "Split atmospheric tomography using laser and natural guide stars," $J$. Opt. Soc. Am. A 25(10), 2427-2435 (2008).

[9] Gilles, L., Wang, L., and Ellerbroek, B. L., "Minimum variance split tomography for laser guide star adaptive optics," European Journal of Control 17(3) (2011).

[10] Ellerbroek, B. L. and Rigaut, F., "Methods for correcting tilt anisoplanatism in laser-guide-star-based multiconjugate adaptive optics," J. Opt. Soc. Am. A 18(10), 2539-2547 (2001).

[11] Noll, R. J., "Zernike polynomials and atmospheric turbulence," J. Opt. Soc. Am. A 66, 207-211 (1976).

[12] Ragazzoni, R., Marchetti, E., and Rigaut, F., "Modal tomography for adaptive optics," Astronomy and Astrophysics 342, L53-L56 (Feb. 1999).

[13] Clare, R. M., Ellerbroek, B. L., Herriot, G., and Véran, J.-P., "Adaptive optics sky coverage modeling for extremely large telescopes," Appl. Opt. 45, 8964-8978 (Dec 2006).

[14] Véran, J.-P. and Herriot, G., "Type II Woofer-Tweeter Control for NFIRAOS on TMT," Adaptive Optics: Methods, Analysis and Applications, JTuC2, Optical Society of America (2009).

[15] Véran, J., Irving, C., Beauvillier, A., and Herriot, G., "Implementation of type-II tip-tilt control in NFIRAOS with woofer-tweeter and vibration cancellation," in [Proc. of the SPIE], 7736 (July 2010).

[16] Correia, C. and Véran, J., "Woofer-tweeter temporal correction split in atmospheric adaptive optics," Submitted to Opt. Lett. (Apr 2012).

[17] Correia, C., Raynaud, H.-F., Kulcsár, C., and Conan, J.-M., "On the optimal reconstruction and control of adaptive optical systems with mirroir dynamics," J. Opt. Soc. Am. A 27, 333-349 (Feb. 2010).

[18] Correia, C., Véran, J.-P., and Herriot, G., "Advanced vibration suppression algorithms in adaptive optics systems," J. Opt. Soc. Am. A 29, 185-194 (Mar 2012).

[19] Kulcsár, C., Raynaud, H.-F., Petit, C., and Conan, J.-M., "Optimal AO control with NGS/LGS wavefront sensors: the multirate case," Adaptive Optics Systems II 7736(1), 773614, SPIE (2010).

\footnotetext{
${ }^{*}$ https://github.com/lianqiw/maos
} 